\title{
Bayesian inference of agent-based models: a tool for studying kidney branching morphogenesis
}

\author{
Ben Lambert ${ }^{1}$ (D) Adam L. MacLean ${ }^{2,3}$. \\ Alexander G. Fletcher ${ }^{4,5}$ - Alexander N. Combes ${ }^{6,7}$. \\ Melissa H. Little ${ }^{7,8}$. Helen M. Byrne ${ }^{2}$
}

Received: 12 July 2017 / Revised: 2 January 2018 / Published online: 1 February 2018

(C) The Author(s) 2018. This article is an open access publication

\begin{abstract}
The adult mammalian kidney has a complex, highly-branched collecting duct epithelium that arises as a ureteric bud sidebranch from an epithelial tube known as the nephric duct. Subsequent branching of the ureteric bud to form the collecting duct tree is regulated by subcellular interactions between the epithelium and a population of mesenchymal cells that surround the tips of outgrowing branches. The mesenchymal cells produce glial cell-line derived neurotrophic factor (GDNF), that binds with RET receptors on the surface of the epithelial cells to stimulate several subcellular pathways in the epithelium. Such interactions are known to be a prerequisite
\end{abstract}

Ben Lambert and Adam L. MacLean have contributed equally to this work.

Electronic supplementary material The online version of this article (https://doi.org/10.1007/s00285018-1208-z) contains supplementary material, which is available to authorized users.

$\triangle \quad$ Ben Lambert

ben.c.lambert@gmail.com

1 Department of Zoology, University of Oxford, Oxford, UK

2 Mathematical Institute, University of Oxford, Andrew Wiles Building, Woodstock Road, Oxford, UK

3 Present Address: Department of Mathematics, University of California, Irvine, Irvine, CA, USA

4 School of Mathematics and Statistics, University of Sheffield, Hicks Building, Hounsfield Road, Sheffield S3 7RH, UK

5 Bateson Centre, University of Sheffield, Firth Court, Western Bank, Sheffield S10 2TN, UK

6 Department of Anatomy and Neuroscience, University of Melbourne, Melbourne, VIC 3010, Australia

7 Murdoch Childrens Research Institute, Flemington Rd, Parkville, Melbourne, VIC 3052, Australia

8 Department of Paediatrics, University of Melbourne, Melbourne, VIC 3010, Australia 
for normal branching development, although competing theories exist for their role in morphogenesis. Here we introduce the first agent-based model of ex vivo kidney uretic branching. Through comparison with experimental data, we show that growth factor-regulated growth mechanisms can explain early epithelial cell branching, but only if epithelial cell division depends in a switch-like way on the local growth factor concentration; cell division occurring only if the driving growth factor level exceeds a threshold. We also show how a recently-developed method, "Approximate Approximate Bayesian Computation", can be used to infer key model parameters, and reveal the dependency between the parameters controlling a growth factor-dependent growth switch. These results are consistent with a requirement for signals controlling proliferation and chemotaxis, both of which are previously identified roles for GDNF.

Keywords Organogenesis - Cellular automaton - Developmental processes · Mathematical modelling $\cdot$ Morphology

Mathematics Subject Classification 92B05

\section{Introduction}

The kidney is a complex organ with a highly branched structure. Its primary function is to filter urea and other waste products from the blood and metabolic system. Kidney and urinary tract congenital disorders are amongst the most common birth defects, with many of these conditions being caused by incomplete branching during development (Airik and Kispert 2007). To understand how such disorders arise, we must first address fundamental questions about the developing kidney, including how branching is initiated, how it is regulated, and how cessation of branching is controlled. These processes are complex and, as such, biological experiments that aim to answer these questions are technically difficult to conduct. Furthermore, each experiment typically investigates only a single facet of kidney morphogenesis. Mathematical and computational models can assist in this endeavour by integrating multiple biological hypotheses via a well-defined set of assumptions. Simulations of the resulting models can be performed to test alternative hypotheses about the mechanisms that regulate kidney morphogenesis, and generate new predictions that can be tested experimentally (Cebrian et al. 2014; Combes 2015; Murray et al. 1983; Packard et al. 2013; Short et al. 2014; Zubkov et al. 2015).

Nephrons are the primary functional units of a kidney. A fully developed human kidney has between 0.2 and 1.8 million nephrons (Hughson et al. 2003), that are connected by a system of collecting ducts to the ureter and bladder (Costantini 2006). Most of the organ is formed during embryonic development, beginning 5 weeks post-gestation in humans and embryonic day 10.5 (E10.5) in mice (Carlson 2013; Cebrian et al. 2004). An outgrowth of epithelial cells from the nephric duct leads to the formation of the ureteric bud epithelium (Little 2015). As the ureteric bud grows and branches, a cloud of mesenchymal progenitor cells form caps at the tips, and eventually differentiate into nephrons. 
In addition to their role as nephron precursors, mesenchymal cells release cytokines that influence the growth and branching of epithelial cells as they form the collecting tree structure. Glial cell line-derived neurotrophic factor (GDNF) is one such growth factor. GDNF is expressed in the metanephric mesenchyme by E11.5, and is thought to diffuse across to the epithelial cell layer, where its binding to cell surface RET receptors transduces signals that are essential for morphogenesis (Durbec et al. 1996). GDNF is known to be a chemoattractant, and also to stimulate the outgrowth of epithelial cells (Little and McMahon 2012). However, there is no consensus about whether these mechanisms are sufficient to generate normal branching, or whether additional chemical or mechanical mechanisms are needed. For example, GDNF-independent signals involving members of the fibroblast growth factor family and Activin are known to affect branching (Maeshima et al. 2007; Michos et al. 2010; Miyazaki et al. 2000; Qiao et al. 2001; Tee et al. 2010, 2013). Additionally, many of these other growth factors have chemical pathways that are intrinsically linked to those of GDNF, meaning that their growth mechanisms may not be entirely independent. As the roles of GDNF and other growth factors remain to be fully elucidated, in this paper we focus on a single generic growth factor which we term "GDNF" and attribute to it actions and effects that we acknowledge are likely caused by a combination of different factors. Although the mechanical forces between epithelium and mesenchyme may influence branching patterns, they are not a prerequisite for branching. Indeed experiments performed by Qiao et al. (1999) have revealed that cell-cell contact between mesenchyme and epithelium is not required for branching; exposure of epithelial cells to soluble factors derived from metanephric mesenchyme is sufficient. It is also possible that mechanical forces between the epithelial cells may be an important mechanism for branching and, indeed, experimental and mathematical modelling work has demonstrated that this mechanism can have a significant effect on branching morphogenesis (Varner and Nelson 2014).

Branching morphogenesis is a characteristic feature of many mammalian organs including the kidney, lung, vasculature, the saliva and mammary glands, and in limb development (Affolter et al. 2009). In each of these tissues, branched structures arise due to repetition of three cellular motifs: bud formation followed by bud extension, and bud splitting, with these processes being facilitated by regulation at the tips and stalks of individual buds. Similar pathways and network topologies perform this regulation in vastly different systems; for example, fibroblast growth factor signalling in combination with Delta/Notch signals regulate branching of both trachea and vertebrate vasculature (Affolter et al. 2009; Ochoa-Espinosa and Affolter 2012). Several alternative theories have been proposed to explain how such signalling generates branched structures. For example, Turing's reaction-diffusion mechanism can give rise to branched structures (Menshykau and Iber 2013). Mechanical theories have also been proposed (Varner and Nelson 2014). To distinguish between competing theories, simple systems are needed, whose variability can be controlled. Explant models of branching have a valuable role to play here as they can recapitulate branching morphogenesis, and are amenable to image analyses that are difficult or impossible to perform in vivo (Basson et al. 2006; Serls et al. 2005; Watanabe and Costantini 2004). Additionally, it is possible not only to monitor the structure of an 
explant as it evolves over time but also to determine for example how these dynamics change when the composition of the culture medium that bathes the explant is altered.

A variety of mathematical models have been developed to study different aspects of branching morphogenesis, including physical cellular processes, such as proliferation and migration (Hirashima et al. 2009), the underlying molecular processes (Zubkov et al. 2015), or a combination of the two (Adivarahan et al. 2013; Clément and Mauroy 2014; Menshykau and Iber 2013). A recent study used ordinary differential equations to describe how the dynamics of the growing epithelial cell populations at the tips of branches are regulated by mesenchymal cells (Zubkov et al. 2015). Comparison of simulation results and experimental data revealed that the mathematical model could recapitulate the observed dynamics for the ratio of epithelial to mesenchymal cells at the branch tips. In other studies a Turing system (Turing 1952) involving the diffusion of the secreted ligands GDNF (produced by mesenchyme) and Wnt11 (produced by the epithelium in response to the presence of GDNF), as well as RET receptors, is proposed to be the primary driver of branching (Menshykau and Iber 2013; Clément and Mauroy 2014). Predictions generated by these models include the need for cooperative receptor-ligand binding (Menshykau and Iber 2013), and a mechanism for self avoidance (so that growing tips do not come into contact) (Clément and Mauroy 2014), which recent experiments suggest may be orchestrated by signalling via BMP7 that is produced by neighbouring branches (Davies et al. 2014).

Whilst a few models of developmental processes consider individual cell behaviour (McLennan et al. 2012, 2015; Fletcher et al. 2014), the majority treats cell populations as a continuum (Clément and Mauroy 2014; Menshykau and Iber 2013; Scialdone et al. 2013; Zubkov et al. 2015). While these models have generated valuable insight into different aspects of organ development, they are limited in their ability to investigate the influence of cellular and subcellular mechanisms. Agentbased frameworks that model individual cell behaviour can offer significant advantages here.

In a biological context, agent-based models treat cells as individual agents, whose behaviour is specified by a predefined set of rules that can be deterministic and/or stochastic. First developed to study the dynamics of replication (von Neumann 1966), simple agent-based models such as cellular automata (CA), and generalisations of these such as cellular Potts and hybrid models are now used widely to study a range of biological systems that include biochemical reaction networks, stem cell proliferation and differentiation, tumour angiogenesis and metastasis (Alarcón et al. 2003; Gerlee and Anderson 2015; Macklin et al. 2012; Roeder et al. 2006; Scott et al. 2014). Such "lattice-based" models restrict agents to sites on a fixed lattice. As such they are typically simpler in design and faster to simulate than alternative off-lattice models, which place fewer restrictions on the movement of cells (or agents) (Bentley et al. 2009; Pathmanathan et al. 2009; Perfahl et al. 2016).

In this paper we investigate a series of experiments performed by Watanabe and Costantini (2004) in which kidneys from mouse embryos were grown in culture. We develop an agent-based framework to model the growth of these explants, and focus on interactions between epithelial cells and growth factors (referred to as "GDNF") 
present in the culture medium. Since the experimental data we have available is limited in detail we formulate an idealised model that contains the minimal number of assumptions necessary to recapitulate key branching features. Whilst the model does not explicitly account for mesenchyme cells, local levels of the generic growth factor, which we term GDNF, serve as a proxy for their influence. We use a CA approach which does not require specific assumptions about the nature of cell-cell forces that cannot (as yet) be experimentally verified. In more detail, our agent-based epithelial cells reside on a regular, two-dimensional grid, their rates of migration and proliferation being regulated by (and, in turn, regulating) the local distribution of GDNF. The length scale of our computational agents is considerably smaller than biological agents, yet we believe that, based on the assumptions which we make, the computational agents' behaviour qualitatively mimics their biological counterparts. When not made explicit, readers should understand that we mean "computational agents" whenever we refer to "agents" or "epithelial cells".

Typically the parameters of computational models cannot be directly measured; they must be inferred from experimental data. Inference can be comparatively expensive in agent-based models, with the stochastic nature of the models requiring multiple simulations for each choice of parameter values. This cost can preclude parameter estimation even with state-of-the-art techniques, such as approximate Bayesian Computation (ABC) (Johnson et al. 2014a; Liepe et al. 2014; Toni et al. 2009). ABC compares simulations from a model with experimental data, and - using statistics that summarise the system behaviour-accepts simulations (and the parameters that generated them), if the statistics for model and data lie within an acceptable distance threshold (see for example, Johnston et al. 2014b; Vo et al. 2015). ABC relies on the ability to simulate a model relatively quickly (typically faster than one second per one stochastic realisation of the model). In situations where this condition is not met, new methods are required for parameter inference. One such method is "Approximate Approximate Bayesian Computation" (AABC) (Buzbas and Rosenberg 2015). With AABC actual model simulations are used to generate pseudo-replicates of the model that can be compared with the data. Since these pseudo-data are generated in a fraction of the time needed to perform real simulations, this method can exhibit significant speed up compared to ABC. Our agent-based CA model takes approximately a minute to generate a single simulation on a desktop computer, and hence we chose to use $\mathrm{AABC}$ for parameter inference.

We analyse the model first by direct simulation across a range of parameter regimes, in each case comparing model outputs and experimental data. We next use AABC to quantitatively fit the model to data, and reveal its dependence on key parameters. In doing so, we show how AABC can be used to integrate the agent-based model (ABM) and the experimental data to answer the following questions:

1. Does the mechanism of GDNF-dependent cellular growth (that is, where cell proliferation rates increase with increases in the local concentration of GDNF) lead kidney explants to develop branches as seen ex vivo?

2. Which characteristics of GDNF-mediated mechanisms are necessary to generate these branches?

3. How sensitive is the model to changes in those parameters that influence branching? 

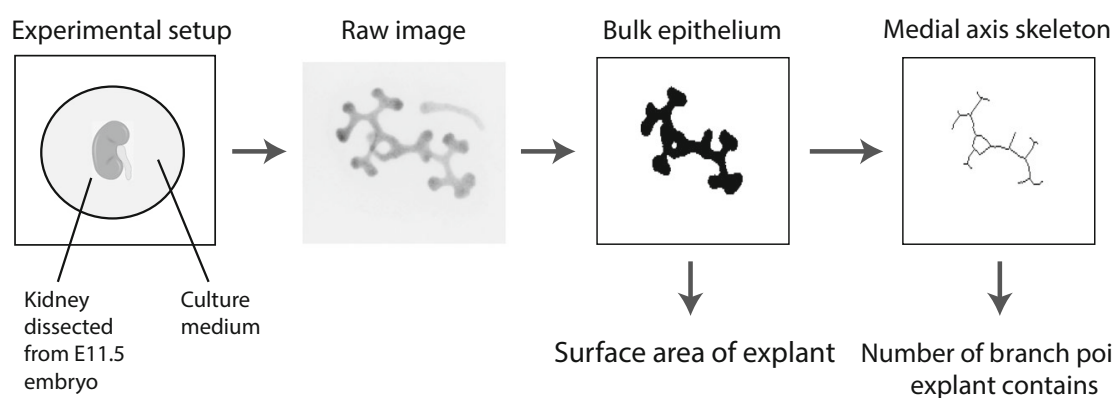

Surface area of explant Number of branch points explant contains
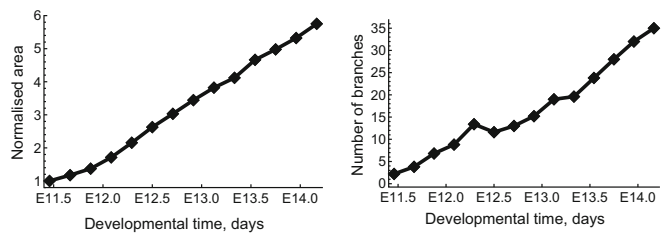

Fig. 1 Workflow used to extract data from the explant images. The same process is applied to simulation results generated from the $\mathrm{CA}$ model. The raw image is reproduced with permission from Watanabe and Costantini (2004)

We now briefly outline the structure of the paper. In Sect. 2.1 we introduce the experimental data that we use in this study, and explain the details of how they are processed to yield summary statistics that represent key features of explant branching. In Sect. 2.2 we describe our CA model and, in doing so, explain how individual cell behaviour depends on a field of GDNF that diffuses through the simulation domain. In Sect. 2.3 we explain how we implemented the AABC method, and then use it to investigate the factors that affect explant branching. In Sect. 3.1 we demonstrate how our model is able to recapitulate the branching of kidney explants, and in Sect. 3.2 we use direct simulation to determine how branching of our model explants depends on specific biological processes. Finally in Sect. 3.3 we use AABC to quantitatively investigate how explant branching depends on three model parameters that quantify the rate of cell motility and cell division.

\section{Materials and methods}

\subsection{Kidney explant data experimental data and image processing}

Watanabe and Costantini (2004) have developed an ex vivo assay to study epithelial branching in murine embryonic development. Kidneys were dissected from E11.5 embryos from a Hoxb7/EGFP transgenic line expressing green fluorescent protein (GFP) throughout the nephric duct and the ureteric bud (Srinivas et al. 1999). Kidneys were cultured in fetal bovine serum and imaged every 30 min over a period of $96 \mathrm{~h}$, generating movies of the developing explants.

We processed videos of the experimental data (three explants in total) by extracting the area of the epithelial mass and the number of branch points in each frame. All image processing and analyses were performed using Matlab (MathWorks, Natick, MA). We 
started by extracting the bulk epithelial mass and then used this to calculate the medial axis skeleton (see Figs. 1, 3): this can be derived as the loci of centres of bi-tangent circles that fit entirely within the epithelial region (Lee 1982). We then counted the number of branch points on each skeleton and used this as a measure of the amount of branching that had occurred. Calculation of the medial axis skeleton and counting of branching points were carried out using Matlab's "bwmorph" function, together with a third-party Matlab package (available from https://uk.mathworks.com/matlabcentral/ fileexchange/11123-better-skeletonization"). While this summary statistic captures the aggregate number of branches, it does not explain other features such as whether the branches are primary or secondary, nor does it provide information about the tipto-stalk ratio (Bush et al. 2014; Little 2015). However we consider the collection and analysis of such detailed summary statistics to be overly sophisticated for comparison with our CA model and, hence, we postpone this for future work.

For comparison with our model simulations we also monitored the area of the bulk epithelium as a measure of the change in the number of cells over time. To facilitate comparison of the data from each experiment we normalised the area of each epithelial mass at time $t$ by its initial mass (at $t=0$ ).

For each CA simulation (described below) we recorded the positions of all epithelial cells over time. We then applied the same image processing methods to the CA data as were applied to the experimental data, in order to generate comparable summary statistics for branching and cell numbers.

\subsection{Cellular automaton model development}

Our model comprises a two-dimensional cellular automaton (CA) model in which the behaviour of individual epithelial cells (i.e. their speed and direction, their rate of division and consumption of GDNF) depends on the locations of nearby cells and the local concentration of GDNF. Cell-cell interactions are governed by rules chosen to replicate observed behaviour. Our model is admittedly an idealisation of the biological processes that underpin kidney morphogenesis. In the absence of suitable experimental data, our model does not explicitly include mesenchyme cells even though they likely surround the explanted epithelium (they are invisible in the experimental images). Similarly our model does not account for a GDNF-Wnt11 positive feedback loop: GDNF secreted by mesenchymal cells binds to RET receptors on epithelial cells and stimulates them to produce Wnt11 which binds to mesenchymal cells, stimulating further uptake of GDNF (Majumdar et al. 2003). However since the available experimental data provides limited information we chose to formulate a simple model that could reproduce key features of kidney morphogenesis.

The epithelial cells occupy a square domain which is discretised into $N \times N$ equally-spaced grid points. Each lattice site is occupied by either an epithelial cell or extracellular matrix (ECM). At $t=0$, a mass of epithelial cells is introduced towards the centre of the domain. Its shape and size (i.e. the number of cells) are chosen to resemble those from the initial images of kidney explants from Watanabe and Costantini (2004). All other sites are occupied by ECM at $t=0$. 
GDNF field We assume that GDNF binds to receptors on the outer membrane of epithelial cells at rate $K_{G}$, and diffuses from the boundaries of the grid, where it is maintained at a constant GDNF concentration, $G_{\infty}$. This implicitly represents a far-field approximation of the experimental conditions, where the ECM area is large, and the local concentration of GDNF is continuously replenished. These conditions are chosen to mimic the effects of GDNF produced by mesenchymal cells (that are likely present) as well as other growth factors that are present in the culture medium. Assuming we adopt previously published estimates of the diffusion coefficient for morphogens (Kicheva et al. 2007; Yu et al. 2009), the time scale for the diffusion of GDNF from the outer border of the mesenchyme to the epithelium (minutes to hours) is much shorter than the time scale for cell division (hours to tens of hours). We exploit this separation of timescales to justify making a quasi-steady state approximation in the $2 \mathrm{D}$ reaction-diffusion equation which describes the distribution of GDNF, $G(x, y, t)$,

$$
\frac{\partial G}{\partial t} \approx 0=D_{G} \nabla^{2} G-\Phi_{G}
$$

where $D_{G}$ denotes the assumed-constant diffusion coefficient for GDNF, and $\Phi_{G}$ is the local rate of GDNF consumption,

$$
\Phi_{G}= \begin{cases}K_{G} G(x, y, t), & \text { for an epithelial cell located at grid point }(x, y) \text { at time } t \\ 0, & \text { for ECM. }\end{cases}
$$

In Eq. (2) we assume, for simplicity, that the rate of GDNF consumption depends linearly on the concentration of substrate (with rate parameter $K_{G}$ ). In what follows, it is convenient to recast Eq. (1) in terms of a dimensionless GDNF concentration $g=\frac{G}{G_{\infty}}$, and non-dimensional spatial parameters, $\eta_{x}=\frac{x}{L}, \eta_{y}=\frac{y}{L}$, where $0 \leq g \leq 1$ is the GDNF concentration as a ratio of that at the boundaries, and $0 \leq \eta_{x, y} \leq 1$ are spatial coordinates as a fraction of the simulation domain size. Equation (1) can then be written as,

$$
d_{g} \nabla_{\eta}^{2} g=\phi_{g}
$$

where $\nabla_{\eta}^{2}$ is the Laplacian with respect to the non-dimensional spatial coordinates, $\eta ; d_{g}=\frac{D_{G}}{K_{G} L^{2}}$ is the non-dimensional diffusion coefficient; and $\phi_{g}=\frac{\Phi_{G}}{K_{G} G_{\infty}}$ is the non-dimensional GDNF uptake term. In these coordinates the boundary conditions are specified as,

$$
\begin{aligned}
& g\left(0, \eta_{y}, t\right)=1=g\left(L, \eta_{y}, t\right), \\
& g\left(\eta_{x}, 0, t\right)=1=g\left(\eta_{x}, L, t\right),
\end{aligned}
$$

and the local rate of GDNF consumption is given by,

$$
\phi_{g}= \begin{cases}g\left(\eta_{x}, \eta_{y}, t\right), & \text { for epithelium at grid point }\left(\eta_{x}, \eta_{y}\right) \text { at time } \mathrm{t} \\ 0, & \text { for ECM. }\end{cases}
$$




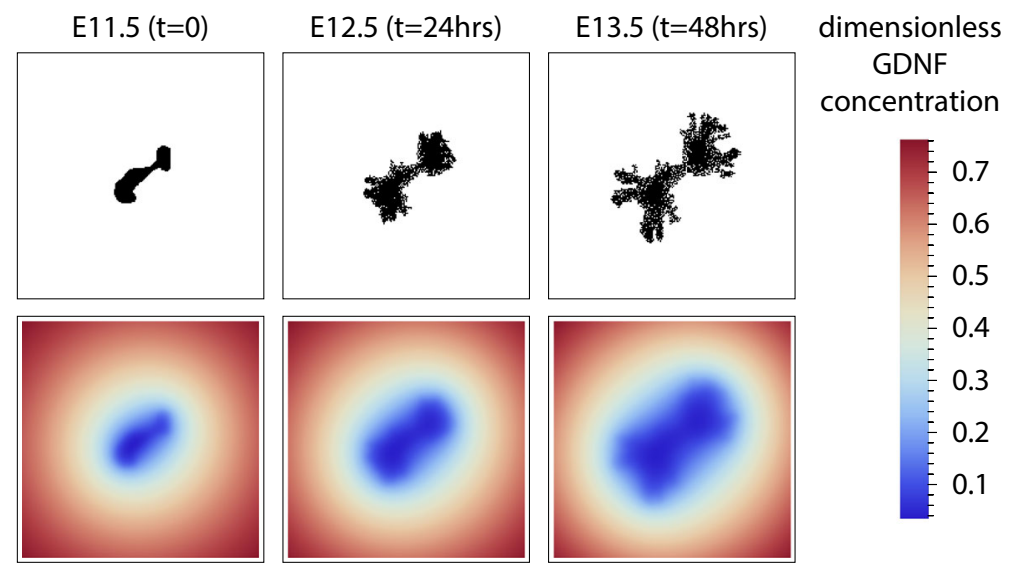

Fig. 2 A series of plots from a typical CA simulation illustrating the interaction between epithelial cell positions (top) and GDNF level (bottom). Note: the GDNF level at the edges is not $g_{\infty}=1$ because the panels only show the central $140 \times 140$ grid points of the domain (itself of size $400 \times 400$ ). The parameter values used to generate these plots are shown in Table S1

In the absence of experimental estimates for the parameters $D_{G}$ and $K_{G}$, preliminary numerical experiments revealed that fixing $d_{g}=0.006$ lead to sufficient variation in GDNF across the spatial domain for branching to occur. (See Table S1 for a summary of the parameter values used in each simulation.)

At the end of each time step (see "Cell based rules" section) we solved Eqs. (3-5) using the method of explicit finite differences (implemented in Matlab). Both the CA model and the finite difference scheme are implemented on the same discrete grid, so the local GDNF level used in the update rules is its value at the grid point where the cell is located. The GDNF field affects the movement and behaviour of epithelial cells, while their location and rates of GDNF uptake affect the evolution of the GDNF field (Fig. 2).

Cell-based rules Simple rules are used to determine whether individual cells move or divide (see Algorithm 1, and Table S1 for the parameter values used); cell death is assumed to be negligible in line with previous experimental results (Hartman et al. 2007). The algorithm begins by solving for the steady state solution of the reactiondiffusion equation (as described above) for the current epithelial cell locations. Using a randomly-permuted list of cell indices, each cell is then visited in turn, and updated. Updates proceed as follows. If a cell has empty neighbouring sites then we determine whether to propose a movement or division event into one of the empty locations; the local GDNF concentration determines whether the proposed action occurs and, if so, the empty site at which the action is carried out.

During this process we establish whether any of the 4 adjacent sites in the von Neumann neighbourhood (up, down, left, right) are empty (no epithelium present). If this is the case then we select a move with probability $p_{\text {move }}$, or a cell division with probability $\left(1-p_{\text {move }}\right)$. The probability $p_{\text {move }}$ is independent of GDNF, and is used simply to propose an action (that may not be undertaken). If an action is selected and feasible, our cell-based rules dictate whether it is executed. Moves are always carried 
out; whether cell division occurs depends on local levels of GDNF. We refer to this process as "GDNF-stimulated cell division". In particular, the probability $p_{c d}$ that a cell at location $\left(\eta_{x}, \eta_{y}\right)$ at time $t$ divides is given by,

$$
p_{c d}=\Phi\left(c_{1}+c_{2} g\left(\eta_{x}, \eta_{y}, t\right)\right)=\frac{1}{\sqrt{2 \pi}} \int_{-\infty}^{c_{1}+c_{2} g\left(\eta_{x}, \eta_{y}, t\right)} e^{-\frac{\tau^{2}}{2}} \mathrm{~d} \tau
$$

where $\Phi($.$) is the standard normal cumulative distribution function. This functional$ form allows a switch-like behaviour for cell division. In Eq. (6) the parameter $c_{1}$ controls the location of the switch, while the parameter $c_{2}$ determines its sensitivity to the local GDNF level, $g(x, y, t)$. In most simulations we fix $c_{1}<0$ and $c_{2}>0$, so that in the absence of GDNF ( $g=0$ ) by default epithelial cells do not divide.

If a move or cell division event is to occur, we must decide which empty neighbouring grid point will be occupied by the result of the action. This choice (if there is more than one empty grid point) is biased by GDNF levels at the neighbouring sites. For moves this process represents "chemotaxis" and for cell divisions it represents "anisotropic cell division". In either case the probability $p_{i}$ of selecting an empty neighbouring grid point $1 \leq i \leq K$ is given by,

$$
p_{i}=\frac{\exp \left(\beta_{a} g_{i}\right)}{\sum_{j=1}^{K} \exp \left(\beta_{a} g_{j}\right)}
$$

where $a \in$ \{move, cell-division $\}$ specifies the action type, $1 \leq K \leq 4$ is the number of empty neighbours, and $g_{i}$ is the GDNF concentration at site $i$. In Eq. (7) the nonnegative parameter $\beta_{a}$ controls the sensitivity of the selection to GDNF concentration, which can differ for chemotaxis and anisotropic cell division.

\subsection{Parameter inference for agent-based models}

Approximate Bayesian Computation allows parameter inference and model selection using distance-based criteria to compare simulations and data (Sunnåker et al. 2013). A distance metric is introduced (e.g. Euclidean or Manhattan) and simulations are rejected if they yield values for a (set of) chosen statistic(s) that exceed a threshold value. $\mathrm{ABC}$ is appropriate when a likelihood function is difficult or intractable to calculate. It has been applied to a wide range of problems in systems biology (Beaumont et al. 2002; Beaumont 2010; Csilléry et al. 2010a). A prerequisite of ABC is that simulating a dataset from the model must be relatively inexpensive so that it possible to adequately sample from the posterior parameter space. This is the case whether performing simple $\mathrm{ABC}$ rejection or more sophisticated procedures, such as sequential Monte Carlo ABC (Toni et al. 2009). A simulation time for a single dataset of $\mathcal{O}$ (seconds) or greater is thus typically prohibitive for performing $\mathrm{ABC}$.

A new algorithm named "Approximate Approximate Bayesian Computation" (AABC) has been designed to resolve this problem (Buzbas and Rosenberg 2015). By replacing true model simulations with realisations of a suitably-tuned statistical 


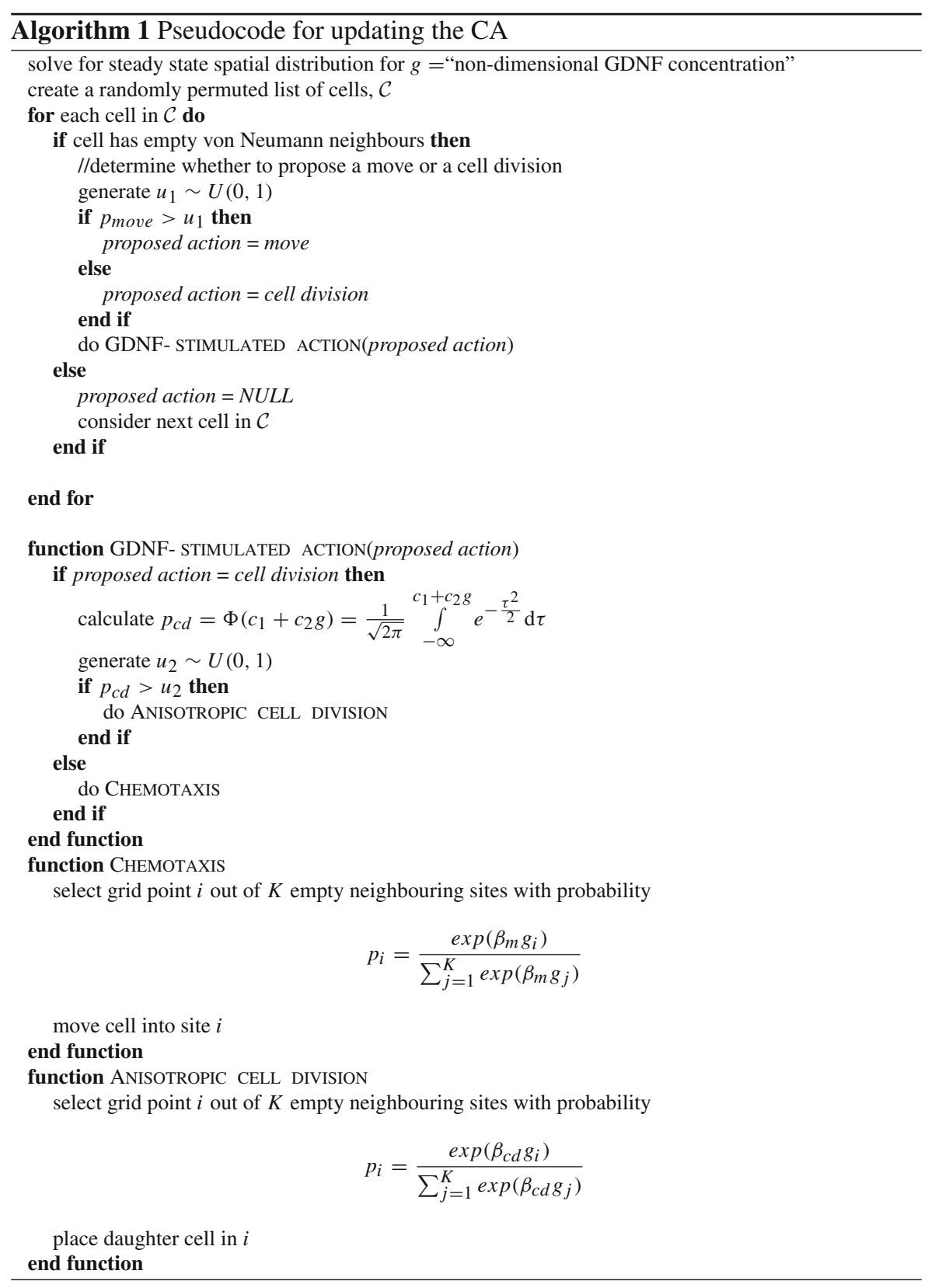


model, AABC enables parameter inference for a class of models that previously presented large (or insurmountable) computational challenges. Agent-based models, such as our CA model, represent such a class, that have with few notable exceptions (Johnston et al. 2014b; Jones et al. 2015; Sottoriva and Tavare 2010), evaded approximate Bayesian inference.

Here we use AABC to infer parameters of the CA model that yield behaviour consistent with the average summary statistics extracted from dynamic experimental data for kidney explants generated by Watanabe and Costantini (2004). These summary statistics (see Sect. 2.1 above) measure two facets of the evolving explants: the number of branches, and the rate of cell proliferation. By comparing summary statistics from the experimental data with equivalent statistics from our simulations (as detailed below) we investigate the sensitivity of explant growth patterns to variation in three model parameters $c_{1}, c_{2}$, and $p_{\text {move }}$. We specify uniform priors for each of these parameters with the following bounds: $c_{1} \in[-20,-40], c_{2} \in[40,280]$ and $p_{\text {move }} \in[0,1]$. The boundaries for the prior distributions of $c_{1}$ and $c_{2}$ were chosen because simulation results indicated that the parameter ranges they encompassed were most likely to yield branching similar to the real explants. Whilst somewhat arbitrary, choosing these parameter ranges was akin to focussing the analysis on the posterior modes, where the majority of posterior probability mass is likely concentrated. All other parameters are held fixed (as per Table S1).

Specifically the algorithm implements the following steps:

1. Simulate the CA model, and accept a subset of particles with parameter sets $\theta_{i}=$ $\left(c_{1 i}, c_{2 i}, p_{\text {movei }}\right)$ and corresponding datasets $\mathbf{x}_{i}=\left(x_{1 i}, x_{2 i}\right), i \in(1,2, \ldots, m)$, where $m$ is the number of simulations (each with a unique set of parameters sampled from the priors) and $N=2$ is the number of replicates per parameter set. For our application, each dataset $x$ consists of data for the normalised area and the number of branch points at $t=10,20,30 \mathrm{~h}$ after E11.5.

2. Sample a new set of parameter values, $\theta^{*}$, from the prior.

3. Calculate the weights, $\omega_{i}$, using an Epanechnikov kernel (Buzbas and Rosenberg 2015):

$$
\omega_{i}=\frac{3}{4} \frac{1}{\left(\theta^{*}-\theta_{(k+1)}\right)}\left[1-\left\|\frac{\theta^{*}-\theta_{i}}{\theta^{*}-\theta_{(k+1)}}\right\|^{2}\right] \mathbb{1}_{\left\{\left\|\theta^{*}-\theta_{i}\right\|<\left\|\theta^{*}-\theta_{(k+1)}\right\|\right\}},
$$

where $\mathbb{1}_{\left\{\left\|\theta^{*}-\theta_{i}\right\|<\left\|\theta^{*}-\theta_{(k+1)}\right\|\right\}}$ is an indicator function with value 1 for the $k$ parameter values $\theta=\theta_{1}, \theta_{2}, \ldots, \theta_{k}$ with the shortest Euclidean distance from $\theta^{*}$, and 0 otherwise (from $\theta_{k+1}$ onwards).

4. Select $\left(\theta_{i}, \mathbf{x}_{i}\right), i \in(1,2, \ldots, k)$, for which $\omega_{i}>0$.

5. Draw a sample $\phi$ - used to specify data resampling probabilities—from a Dirichlet distribution parameterised by $\omega_{i}, i \in(1,2, \ldots, k)$.

6. Simulate a new dataset $\mathbf{x}^{*}$ of area and branching time point data (of size $N=2$ replicates) by: (i) resampling datapoints from $\mathbf{x}_{i}$ with probabilities set by $\phi$, and (ii) assuming that each replicate is equally probable. 


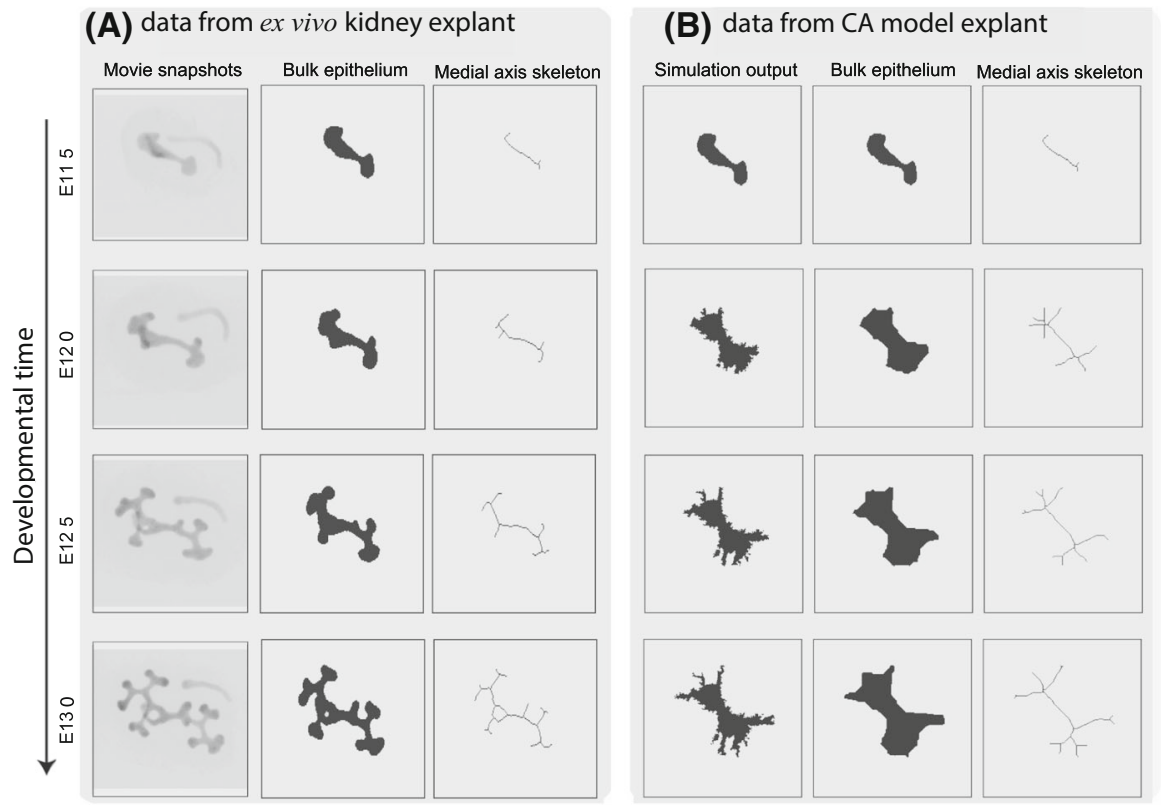

Fig. 3 Comparison of branching patterns associated with $\mathbf{a}$ ex vivo data and $\mathbf{b}$ typical simulations of the CA model. Image processing techniques (see Sect. 2.1) are used first to extract the bulk shape of the epithelial cells (middle panel) and then to generate the medial axis skeleton (right panel), which characterises the shape of the growing explant. The parameter values used to generate these simulation results are shown in Table S1

7. Calculate the Euclidean distance between the real and simulated datasets, and add $\theta^{*}$ to the posterior, iff $\left\|\mathbf{x}_{i}-\mathbf{x}^{*}\right\|<\epsilon$. We choose $\epsilon$ such that $5 \%$ of simulations are accepted as posterior samples.

8. Repeat steps (2)-(7) until convergence in the approximate posterior distribution is reached.

We perform Step (1) of the algorithm in Matlab (MathWorks, Natick, MA), and steps (2)-(7) in Julia (v0.3.5, julialang.org).

\section{Results}

\subsection{GDNF-directed cellular proliferation can explain the branching patterns observed when kidney explants are cultured ex vivo}

Simulations of the CA model reveal that it can generate branching patterns similar to those identified from ex vivo kidney explant data of Watanabe and Costantini (2004). In Fig. 3 we compare results from a typical simulation with experimental data collected at four time points. The CA model recapitulates notable features of branching: branching at both ends of the buds, followed by secondary branching at the branch tips. Our simulated explants also often produce branching events in which three or 
Table 1 A summary of the effect of different GDNF signalling mechanisms on explant branching

\begin{tabular}{llllc}
\hline Figures & $\begin{array}{l}\text { GDNF-stimulated } \\
\text { cell division }\end{array}$ & Chemotaxis & $\begin{array}{l}\text { Anisotropic cell } \\
\text { division (ACD) }\end{array}$ & Branching \\
\hline $4,5 \mathrm{a}$ & - & - & - & - \\
$4,5 \mathrm{~b}$ & + & - & - & + \\
$4,5 \mathrm{c}$ & - & + & - & - \\
$4,5 \mathrm{~d}$ & + & + & - & + \\
$4,5 \mathrm{e}$ & - & - & + & - \\
$4,5 \mathrm{f}$ & + & - & + & ++ \\
$4,5 \mathrm{~g}$ & - & + & + & - \\
$4,5 \mathrm{~h}$ & + & + & + & ++ \\
\hline
\end{tabular}

Each row of the table corresponds to specific panels of Figs. 4 and 5, with corresponding parameter sets indicated in Table S1. In the middle three columns "-" indicates that the mechanism is inactive, "+" indicates that it is active. In the right-hand column "-" indicates no significant branching, "+" indicates modest branching and " ++ " indicates that branching that is consistent with experimental data

more branches emerge from a single tip, events which are observed in the developing kidney (Menshykau and Iber 2013).

\subsection{Finding a minimal set of sub-cellular mechanisms necessary to generate branching}

We also used our model to investigate the contribution of cell proliferation, anisotropic cell division and chemotaxis to branching (Table 1 and Figs. 4, 5). Each mechanism is regulated by local levels of GDNF: the rate of cell division increases with local levels of GDNF in a sigmoidal manner (see Fig. 6); regarding chemotaxis, cells are more likely to migrate up local GDNF gradients; for anisotropic cell division (ACD), daughter cells are located preferentially in sites with higher levels of GDNF. For both chemotaxis and ACD we acknowledge that our model is an idealisation of the biological processes involved. For example RET-dependent movement (Riccio et al. 2016) and luminal mitosis (Packard et al. 2013) are not considered explicitly. Even so, our model captures some of the features of these processes and, hence, can be used to determine their relative contributions for branching.

Model simulations reveal that when cell division is independent of GDNF, no branching occurs; the explant grows as an approximately circular mass (see Figs. 4 , 5a). When only cell proliferation is regulated by GDNF, some branching occurs, although the number of branches is fewer than for explant growth (see Figs. 4, 5b). When chemotaxis and/or anisotropic cell division depend on GDNF although the rate of proliferation is independent of GDNF, the model does not exhibit branching (Figs. $4,5 \mathrm{c}, \mathrm{e}, \mathrm{g}$ ). Additionally the cumulative effect of GDNF-stimulated proliferation and chemotaxis on branching is no greater than proliferation stimulated by GDNF alone (Figs. 4, 5d). By contrast when proliferation and anisotropic cell division depend on GDNF, the number of branches observed along the branching trajectory increases 
such that simulation results are in good agreement with the experimentally observed branching patterns (Figs. 4, 5f). The best agreement with the experimental data is obtained when all three processes (i.e. proliferation, chemotaxis and anisotropic cell division) depend on GDNF levels (Figs. 4, 5h). These results do not appear to depend strongly on the initial shape of the epithelium used to in model simulations. In particular simulations initialised using different experimental explant data yielded similar results (see Figs. S1, S2).

These simulation studies reveal several key messages. First, GDNF-stimulated proliferation can generate biologically realistic branching patterns. Second, what we term anisotropic cell division ameliorates the rate of branching when it is coupled to GDNF-stimulated growth. Finally, within the regions of parameter space studied, GDNF-controlled chemotaxis does not strongly affect the branching of explants. It was an unexpected result that chemotaxis and anisotropic cell division did not play a more significant role in generating branching, but this appears to reflect the fact that, in our model, the movement and location of daughter cells in cell division is often highly constrained, and skewed towards sources of GDNF. In order to determine whether the weak dependence on chemotaxis was due to an overly simplistic treatment of cell-cell interactions, we revised our CA model to ensure that epithelial cells remain attached to at least one neighbouring cell. However this change did not significantly alter the observed branching patterns (results not shown).

We also investigated how the shape of the GDNF-controlled switch that regulates cell proliferation affects branching (Fig. 6). When the slope of the switching function is gradual, the epithelium grows as a circular mass and no branching occurs (Fig. 6a). Similarly, when the threshold of the switch is high, the epithelial growth rate and branching rate are both too low (Fig. 6c). We therefore tune the threshold and slope of the GDNF-mediated switch (Fig. 6b) in order to maximise the amount of branching. Again we show in the Supplementary Materials how these results are robust to variation in the shape of the epithelial cell starting mass (see Figs. S3 and S4).

\subsection{Branching is sensitive to the form of the GDNF proliferation switch}

Having shown that our CA model can reproduce qualitative features associated with early kidney morphogenesis, we now study the dependence of branching characteristics on the parameters $c_{1}, c_{2}$, and $p_{\text {move }} ; c_{1}$ and $c_{2}$ jointly determine the sensitivity of cell proliferation to GDNF levels (see Eq. (6)), and $p_{\text {move }}$ is the probability of cell migration.

We performed 50,000 AABC simulations and accepted the top 5\% (the 5\% with overall smallest Euclidean distances from the summary statistics associated with the experimental data) to compose approximate posterior samples, as outlined in Sect. 2.3. We use two summary statistics to compare simulations with data: the normalised area and the total number of branch points as calculated from medial axis skeletons of the epithelial cell mass.

In Fig. 7a we plot those trajectories that have been accepted as posterior samples. The marginal posterior distributions associated with each parameter are shown on the diagonal of Fig. $7 \mathrm{~b}$ alongside the two-dimensional posterior joint density distributions 
(A)

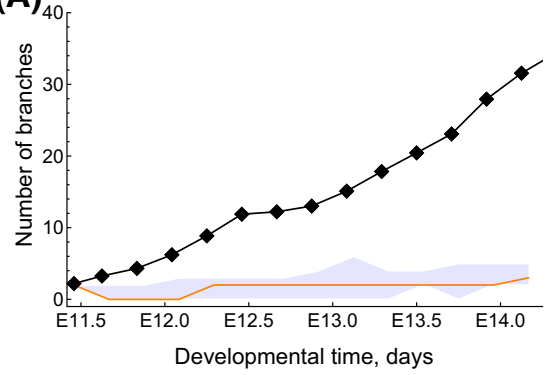

(C)

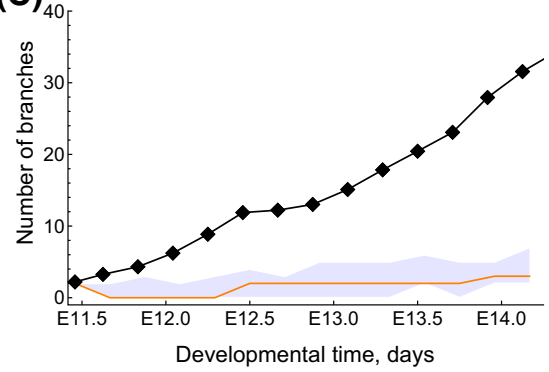

(E)

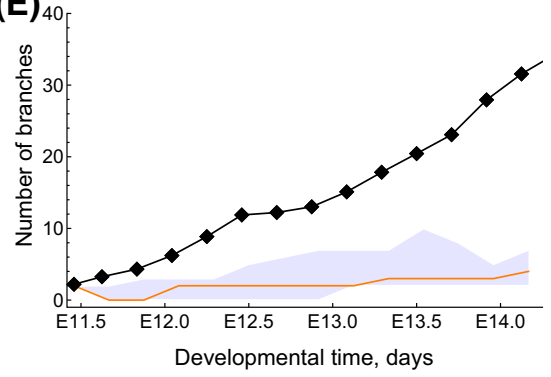

(G)

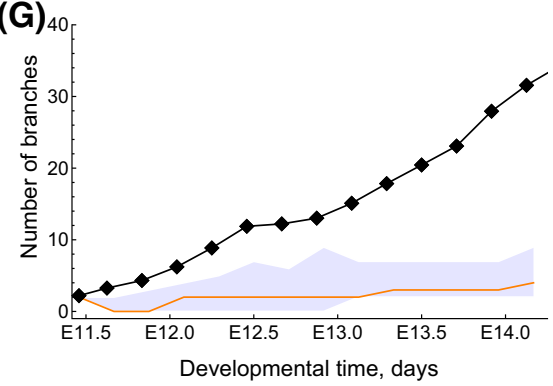

(B)

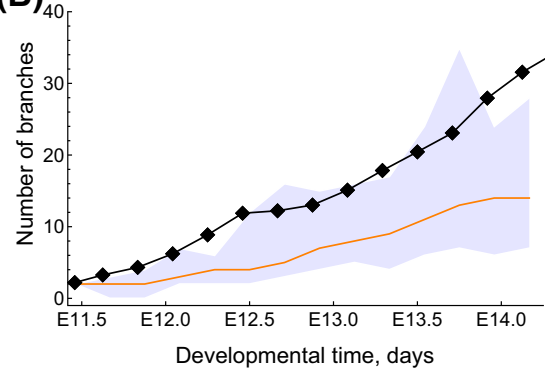

(D)

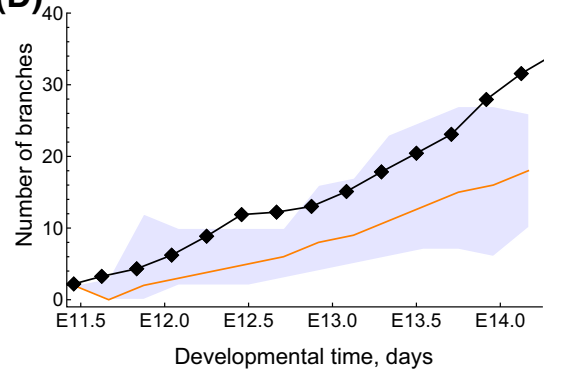

$(\mathbf{F})_{40}$

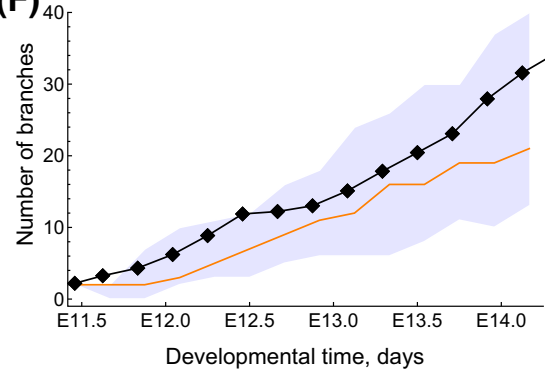

(H)

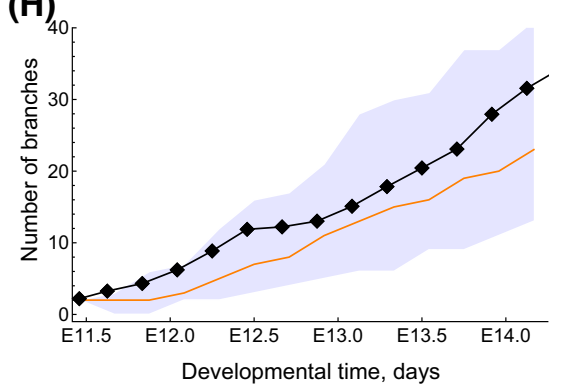

Fig. 4 The effect of different GDNF signalling mechanisms on explant branching. a-h Simulation results with the indicated mechanisms implemented, and correspond to cell distributions shown in the panels of Fig. 5 with the same letters. In each panel the black line and points represent the evolution of branches from an explant experiment in Watanabe and Costantini (2004); the orange line represents the mean branching observed by model simulation $(\mathrm{n}=200)$ and the shaded region indicates the $95 \%$ confidence interval. "ACD" indicates "anisotropic cell division". The parameter values used in each case are in Table S1 (colour figure online) 
(I.) Real explant data

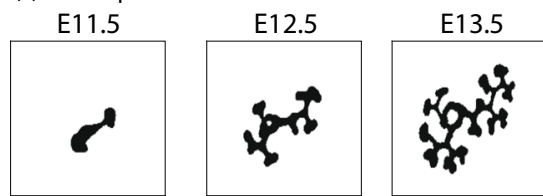

(A) Random proliferation only

(B) GDNF-stimulated proliferation only
E11.5 $\quad$ E12.5
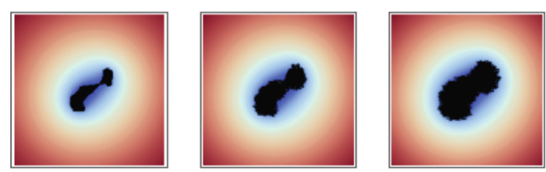

(C) Random proliferation + chemotaxis
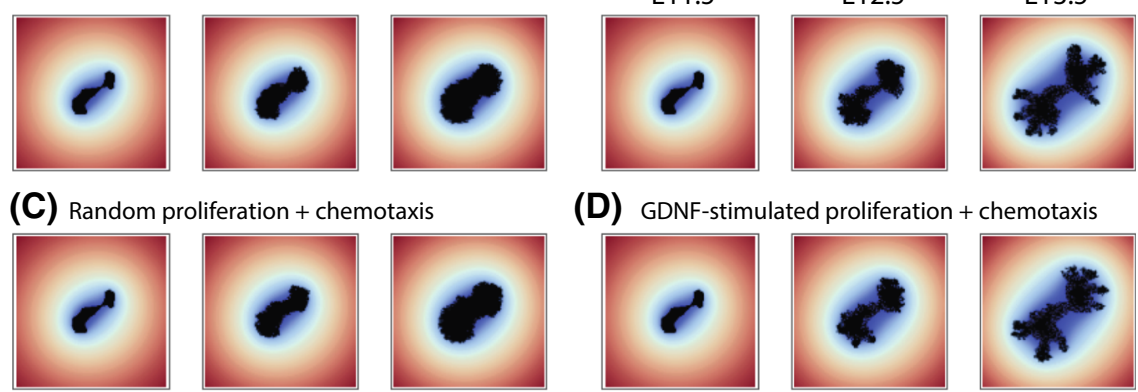

(D) GDNF-stimulated proliferation + chemotaxis
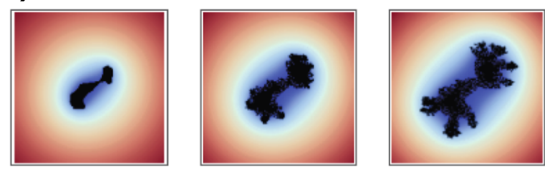

(E) Random proliferation $+A C D$

(F) GDNF-stimulated proliferation + ACD
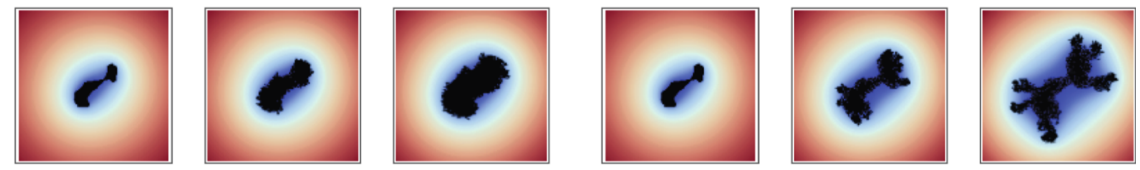

(G) Random proliferation + chemotaxis + ACD
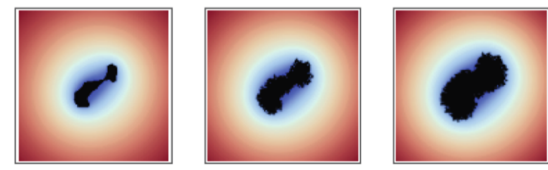

(H) GDNF-stimulated proliferation + chemotaxis + ACD
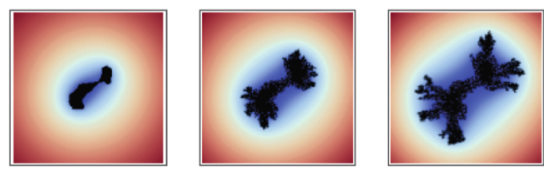

Fig. 5 The effect of different GDNF signalling mechanisms on explant branching. a-h Simulation results with the indicated mechanisms implemented, and correspond to the panels in Fig. 4 with the same letters. The black mass is the epithelial cells, and the coloured shading shows the corresponding dimensionless distribution of GDNF. For comparison experimental from the first video in Watanabe and Costantini (2004) are presented in (I.). "ACD" indicates "anisotropic cell division". Note: the GDNF level at the edges is not $g_{\infty}=1$ because the panels only show the central $140 \times 140$ grid points of the domain (itself of size $400 \times 400$ ). The parameter values used in each case are in Table S1 (colour figure online)

for each pair of parameters. This reveals that the marginal posterior distribution for $c_{2}$ deviates most from its prior, taking values between 40 and 280 with high probability; both $c_{1}$ and $p_{\text {move }}$ deviate less from their prior, although $p_{\text {move }}$ shows deviation at high values where little or no growth occurs.

The joint density plots in Fig. 7b reveal dependencies in parameter space. For the parameter pair $\left(c_{1}, c_{2}\right)$, a negative correlation is observed; we note also that for low values of $c_{1}$ (i.e. $\left.c_{1} \in[-40,-25]\right) c_{2}$ is tightly constrained. This dependence is expected since the two parameters jointly determine the sensitivity of the switch to GDNF. In particular, under a local non-dimensional concentration of GDNF of $g=-\frac{c_{1}}{c_{2}}$ a cell with free neighbouring space divides with probability 0.5 . This means that there are regions of $\left(c_{1}, c_{2}\right)$ space (where $c_{1} \sim-k c_{2}$ ) which result in practically 
(A)

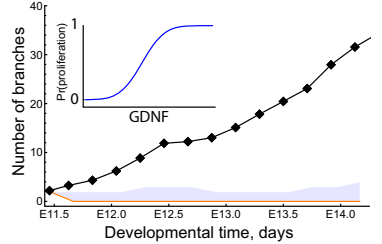

(B)

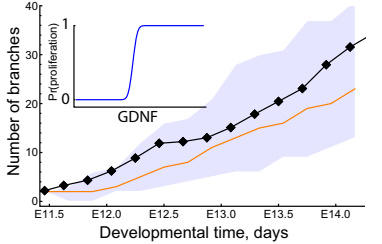

(C)

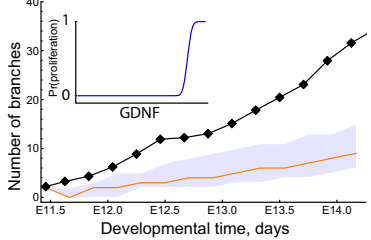

Fig. 6 Series of simulation results showing how the shape of the GDNF-mediated proliferation switch influences the branching dynamics. For the results shown in each of the panels we ran simulations with $c_{1}=-25$ so that in the absence of GDNF cells would not divide, and we vary $c_{2}$ across each of the panels: for $\mathbf{a} c_{2}=400, \mathbf{b} c_{2}=120$ and $\mathbf{c} c_{2}=20$. The inset panels show the location of the proliferation switch in GDNF-space (horizontal axis), against the probability of growth (vertical axis). In each panel the black line and points represent the evolution of branches from an explant experiment in Watanabe and Costantini (2004); the orange line represents the mean branching observed by model simulation $(n=200)$ and the shaded region indicates the $95 \%$ confidence interval. The parameter values used in each case are shown in Table S1 (colour figure online)

identical GDNF switches. For the other two parameter pairs, the joint density plots highlight what has been termed "sloppiness" (Gutenkunst et al. 2007): the explant branching phenotype is robust to changes in the values of $c_{1}$ and $p_{\text {move }}$. Parameter sloppiness can be symptomatic of several problems, including insufficient data, or a model that is overly complex for the available data. Additionally we cannot discount the possibility that our summary statistics are suboptimal, although we believe that the use of more detailed summary statistics may be excessive given the level of biological and mechanical realism present in our CA model.

\section{Discussion}

We have developed a new model to describe branching morphogenesis in the developing kidney. While there are many agent-based models of other branching systems (e.g. Fumoto et al. 2016; Iber and Menshykau 2013; Lindenmayer 1968; Merks and Koolwijk 2009; Schatten et al. 2007) to our knowledge, this is the first agent-based model of kidney morphogenesis. A significant advantage of our approach is that unlike spatiallyaveraged compartment-based methods (e.g. Menshykau and Iber 2013; Zubkov et al. 2015) it allows study of processes at the single-cell level. This spatial resolution may be particularly important in determining the thresholds in the concentration of growth factors at which branching occurs. The $\mathrm{CA}$ framework allows a cell-based description of tissue morphogenesis and facilitates the future addition of other biophysical mechanisms and the subcellular signalling pathways, as well as extension to include other cell types (e.g. mesenchymal cap cells, when considering kidney morphogenesis).

At present there is no consensus about whether diffusion-driven Turing patterns of GDNF coupled with GDNF-regulated proliferation mechanisms can explain branching, or whether other chemical and mechanical mechanisms are required. Our simulation results indicate that the GDNF-mediated proliferation may suffice to generate branching in this system. We find that to recapitulate the branching behaviour of the developing kidney, dynamic spatial patterning of epithelial cell proliferation had to 

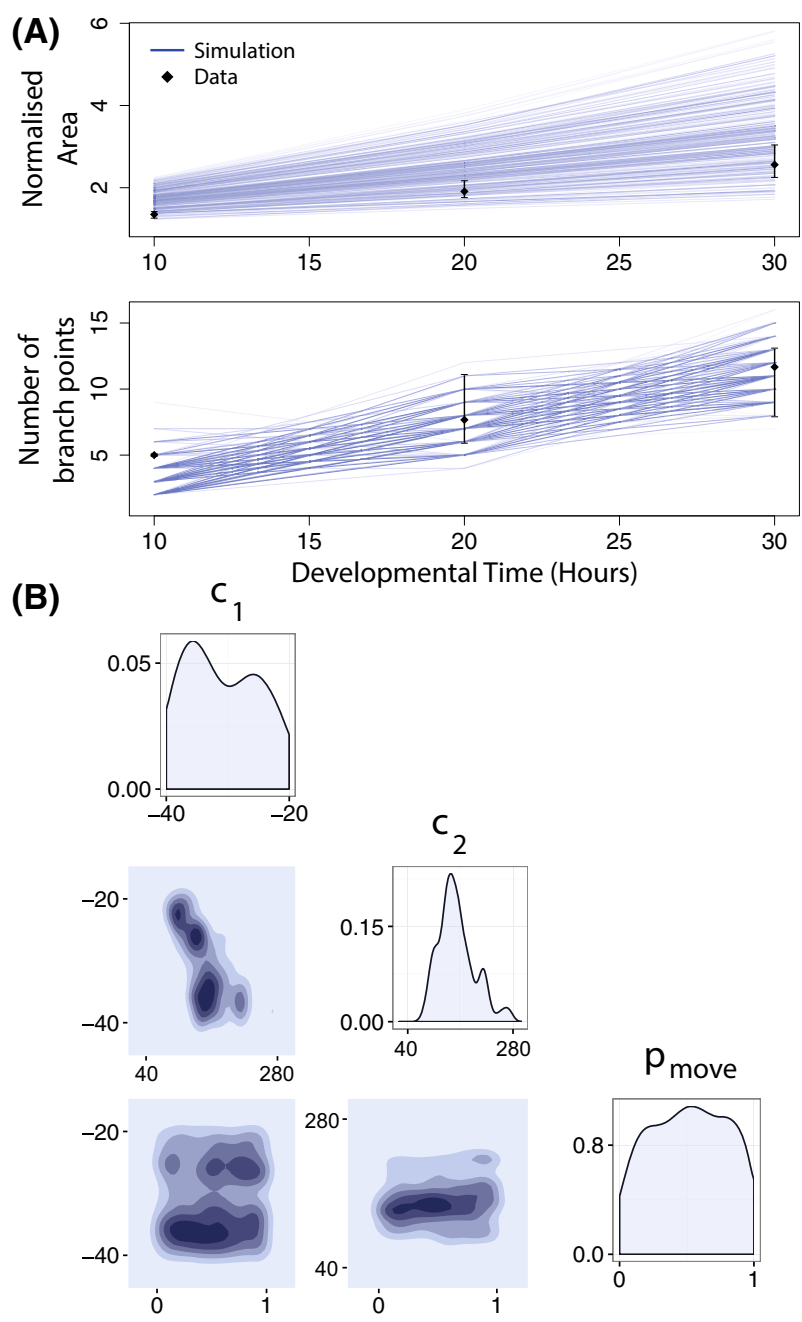

Fig. 7 Approximate posteriors distributions for three model parameters as estimated by AABC. The parameters $c_{1}$ and $c_{2}$ affect the location and sensitivity of the GDNF-mediated growth switch, and $p_{m o v e}$ determines the relative likelihood of cell movement rather than cell division (see Sect. 2). a Accepted trajectories in the posterior simulated from the model and their comparison with the ex vivo data (black dots indicate means, and error bars show the range of the data). The experimental data are the means of summary statistics extracted from three explant experiment videos in Watanabe and Costantini (2004). b Posterior parameter distributions for single (on the diagonal) and joint pairs of parameters. See Table S1 for information about the parameter values used in the simulations

be included in the model. This was the case across all the parameter sets that we considered, however there may be isolated regions in parameter space in which branching occurs via independent mechanisms not included in our model (e.g. due to mechanical cell-cell interactions). Simulation studies demonstrated that GDNF-stimulated tissue growth together with chemotaxis and anisotropic cell division provided the 
best fit to the explant branching data studied. In a recent experimental study, Riccio et al. (2016) studied the behaviour of tip cells across development, and concluded that GDNF signalling largely drives cell movement (rather than proliferation), and that this movement, in turn, drives branching. While GDNF-dependent chemotaxis may play a role later in kidney development (we focus here on E11.5 to E14), our results suggest that it may not contribute significantly during early branching of the epithelium, and supports a greater role for proliferation than that identified by Riccio et al. (2016). It is, of course, possible that mesenchyme-derived factors other than GDNF provide this signal. Inference of the CA model on a three-dimensional subset of parameters revealed dependency between the parameters controlling the GDNF-stimulated cell proliferation switch $\left(c_{1}\right.$ and $\left.c_{2}\right)$. Specifically this result indicated how cell proliferation should depend strongly on GDNF levels. However it remains to identify a biological mechanism that fulfils this criteria.

Whilst our model can generate explant patterns that mimic some aspects of the experimental data, we recognise that our simulation results often differ in the finer details. At present it is not clear whether these discrepancies are due to biological mechanisms that we have neglected, or due to the relatively simple nature of our model framework. For example these differences might be reduced if an off-lattice agent-based model, incorporating cell-cell contact forces, were used. We also note that the differences between our simulation results and the experimental data are highest for later developmental times, and we speculate that other mechanisms may be responsible for continued branching at these later time points. In particular our model does not explicitly include mesenchymal cells that produce growth factors which may influence branching at later developmental times. Another candidate mechanism is heterogeneity in RET expression amongst different epithelial cells (Shakya et al. 2005). In particular, epithelial cells with differing levels of RET expression have been shown to compete for positions within branches (Riccio et al. 2016), and contribute unequally to the tips of the developing kidney (Chi et al. 2009). We also assumed that the time scale for GDNF diffusion is much slower than the time scale for its uptake/binding to RET receptors. However we cannot discount that future experiments may invalidate this assumption.

To compare our simulation results with the experiment results of Watanabe and Costantini (2004), in both cases we calculated the number of explant branches using an image processing method based on estimating a skeletal representation of 2D explant images (known as the medial axis skeleton; Lee 1982). Determining the number of branches of a 3D explant from a 2D image is nontrivial and we recognise that other techniques have also been employed to this task (see, for example, Short et al. 2013), and it would be interesting as future work to compare these approaches with ours. Similarly, it would be worthwhile considering how well our image processing pipeline and resultant summary statistics based on $2 \mathrm{D}$ images captures the branching in a 3D explant (see Grogan et al. 2017 for an example of this type of work applied to computational models of tumours). In future work, additional summary statistics relating, for example, to branch angles could be calculated by Tree Surveyor-software developed specifically to quantify the dynamics of branching morphogenesis (Short et al. 2013). These data could then be fitted to a more detailed model of kidney explants. 
In order to validate a more detailed model against more advanced summary statistics, additional experimental data is required.

As important differences exist between the dynamics of branching as it occurs ex vivo and in vivo (Short et al. 2014), caution should be exercised when extrapolating from the former to the latter. Here we focus on an ex vivo model, due to the availability of published image data. This system also lends itself more naturally to 2D modelling, which is less computationally expensive than 3D simulations. Scaling an $\mathrm{ABM}$ approach to 3D represents a significant computational hurdle, and it is possible that the different topology may require qualitatively different mechanisms for branching to occur. However our results are supported by the results of computational modelling by Clément and Mauroy (2014) who found that diffusion of growth factors was sufficient to generate realistic 3D branching patterns. Further our 2D model permits investigation of the dominant mechanisms in a simpler geometry, and presents an opportunity for a first model validation step before investigating 3D dynamics. As noted above our model omits certain details of the biology, for example, the (likely) presence of a growth factor-producing mesenchyme. This choice was dictated partly by the sparsity of the available experimental data. However since our simple model was able to reproduce key aspects of the explant branching we chose not to include further (uncertain) biological details.

The flexibility that CA models offer in their ability to describe spatiotemporal heterogeneities is not only an advantage but also a limitation, because the biological interpretation of each update rule is not always clear. Modelling cell proliferation and migration with biophysical and mechanical forces could improve the mechanistic understanding of the model, but comes at significant computational cost (see for example, Kim et al. 2007; Rejniak and Anderson 2011).

To perform parameter estimation, we have implemented a version of AABC, an approximate Bayesian inference scheme that is ideally suited to models such as ours that are computationally expensive to simulate. With the ever-increasing resolution of spatiotemporal data and, concurrently, an increasing number of models developed to describe relevant biological phenomena, we propose that $\mathrm{AABC}$ may find useful applications to a range of problems in systems biology, outside the more typical population genetics applications for which it was developed (Beaumont et al. 2002; Buzbas and Rosenberg 2015; Csilléry et al. 2010b).

Existing models of organ development have proposed alternative mechanisms for branching kidney organogenesis. In Zubkov et al. (2015), a spatially-averaged system of ODEs is proposed in which branching occurs at a specific cell ratio of epithelial (tip) and mesenchymal (cap) cells. In both Clément and Mauroy (2014) and Menshykau and Iber (2013), spatially-resolved models are developed and a growth-promoting ligand mechanism is proposed for branching; in Menshykau and Iber (2013), the authors show that this leads to a Turing-type mechanism through interaction of GDNF and the RET receptor. The model that we present is consistent with these results, but goes further by proposing cellular scale rules that, coupled to the influence of a ligand field, enable branching.

We recognise that other modelling frameworks have been used to simulate branching morphogenesis. These include phase field models (Ohta et al. 1989; Hartmann and Miura 2006) and Turing models (Kondo and Miura 2010; Menshykau and Iber 2013)), 
that yield similar conclusions to our CA approach. Even so, we believe that it is important to establish whether results are robust across different modelling approaches or specific to a particular modelling paradigm.

In conclusion we note that while branching morphogenesis is an old problem in mathematical biology (Murray et al. 1983), many open questions remain to be addressed. Here, using an agent-based model that directly describes the cell-cell interactions that occur during organ development, we shed light on the processes involved in defining the structure of the kidney. In the future, we propose that more complex hybrid models that combine biophysical and experimentally-validated rules for the migration and proliferation of epithelial cells will lead to further advances in our understanding of kidney morphogenesis. Additionally we argue that future models of in vivo kidney development should include other cells types known to be involved in organogenesis, for example, mesenchymal cells.

Acknowledgements We thank Joe Pitt-Francis for useful advice on the image-processing of the explant data. We also thank the EPSRC (Grant No. EP/F500394/1) for partial funding.

Open Access This article is distributed under the terms of the Creative Commons Attribution 4.0 International License (http://creativecommons.org/licenses/by/4.0/), which permits unrestricted use, distribution, and reproduction in any medium, provided you give appropriate credit to the original author(s) and the source, provide a link to the Creative Commons license, and indicate if changes were made.

\section{References}

Adivarahan S, Menshykau D, Michos O, Iber D (2013) Dynamic image-based modelling of kidney branching morphogenesis. Computational methods in systems biology. Springer, Berlin, pp 106-119

Affolter M, Zeller R, Caussinus E (2009) Tissue remodelling through branching morphogenesis. Nat Rev Mol Cell Biol 10(12):831-842

Airik R, Kispert A (2007) Down the tube of obstructive nephropathies: the importance of tissue interactions during ureter development. Kidney Int 72(12):1459-1467

Alarcón T, Byrne HM, Maini PK (2003) A cellular automaton model for tumour growth in inhomogeneous environment. J Theor Biol 225(2):257-274

Basson MA, Watson-Johnson J, Shakya R, Akbulut S, Hyink D, Costantini FD, Wilson PD, Mason IJ, Licht JD (2006) Branching morphogenesis of the ureteric epithelium during kidney development is coordinated by the opposing functions of GDNF and Sprouty1. Dev Biol 299(2):466-477

Beaumont MA, Zhang W, Balding DJ (2002) Approximate Bayesian computation in population genetics. Genetics 162(4):2025-2035

Beaumont MA et al (2010) Approximate Bayesian computation in evolution and ecology. Annu Rev Ecol Evol Syst 41(379-406): 1

Bentley K, Mariggi G, Gerhardt H, Bates PA (2009) Tipping the balance: robustness of tip cell selection, migration and fusion in angiogenesis. PLoS Comput Biol 5(10):e1000549

Bush KT, Martovetsky G, Nigam SK (2014) Relevance of ureteric bud development and branching to tissue engineering, regeneration and repair in acute and chronic kidney disease. Curr Opin Organ Transpl 19(2):153-161

Buzbas EO, Rosenberg NA (2015) AABC: approximate approximate Bayesian computation for inference in population-genetic models. Theor Popul Biol 99:31-42

Carlson BM (2013) Human embryology and developmental biology, 5th edn. Saunders, Philadeiphia

Cebrian C, Borodo K, Charles N, Herzlinger DA (2004) Morphometric index of the developing murine kidney. Dev Dyn 231(3):601-608

Cebrian C, Asai N, D'Agati V, Costantini FD (2014) The number of fetal nephron progenitor cells limits ureteric branching and adult nephron endowment. Cell Rep 7(1):127-137 
Chi X, Michos O, Shakya R, Riccio P, Enomoto H, Licht JD, Asai N, Takahashi M, Ohgami N, Kato M, Mendelsohn C, Costantini FD (2009) Ret-dependent cell rearrangements in the Wolffian duct epithelium initiate ureteric bud morphogenesis. Dev Cell 17(2):199-209

Clément R, Mauroy B (2014) An archetypal mechanism for branching organogenesis. Phys Biol 11(1):016003

Combes AN (2015) Towards a quantitative model of kidney morphogenesis. Nephrology 20(5):312-314

Costantini FD (2006) Renal branching morphogenesis: concepts, questions, and recent advances. Differentiation 74(7):402-421

Csilléry K, Blum MGB, Gaggiotti OE, François O (2010a) Approximate Bayesian computation (ABC) in practice. Trends Ecol Evol 25(7):410-418

Csilléry K, Blum MGB, Gaggiotti OE, François O (2010b) Approximate Bayesian computation (ABC) in practice. Trends Ecol Evol 25(7):410-418

Davies JA, Hohenstein P, Chang C-H, Berry R (2014) A self-avoidance mechanism in patterning of the urinary collecting duct tree. BMC Dev Biol 14(1):1

Durbec P, Marcos-Gutierrez CV, Kilkenny C, Grigoriou M, Wartiowaara K, Suvanto P, Smith D, Ponder B, Costantini FD, Saarma M, Sariola H, Pachnis V (1996) GDNF signalling through the Ret receptor tyrosine kinase. Nature 381(6585):789-793

Fletcher AG, Osterfield M, Baker RE, Shvartsman SY (2014) Vertex models of epithelial morphogenesis. Biophys J 106(11):2291-2304

Katsumi Fumoto, Takigawa-Imamura H, Sumiyama K, Kaneiwa T, Kikuchi A (2016) Modulation of apical constriction by Wnt signaling is required for lung epithelial shape transition. Development 144(1):151162

Gerlee P, Anderson ARA (2015) The evolution of carrying capacity in constrained and expanding tumour cell populations. Phys Biol 12(5):056001

Grogan JA, Markelc B, Connor AJ, Muschel RJ, Pitt-Francis JM, Maini PK, Byrne HM (2017) Predicting the influence of microvascular structure on tumor response to radiotherapy. IEEE Trans Biomed Eng 64(3):504-511

Gutenkunst RN, Waterfall JJ, Casey FP, Brown KS, Myers CR, Sethna JP (2007) Universally sloppy parameter sensitivities in systems biology models. PLoS Comput Biol 3(10):1871-1878

Hartmann D, Miura T (2006) Modelling in vitro lung branching morphogenesis during development. J Theor Biol 242(4):862-872

Hartman HA, Lai HL, Patterson LT (2007) Cessation of renal morphogenesis in mice. Dev Biol 310(2):379_ 387

Hirashima T, Iwasa Y, Morishita Y (2009) Dynamic modeling of branching morphogenesis of ureteric bud in early kidney development. J Theor Biol 259(1):58-66

Hughson M, Farris AB, Douglas-Denton R, Hoy WE, Bertram JF (2003) Glomerular number and size in autopsy kidneys: the relationship to birth weight. Kidney Int 63(6):2113-2122

Iber D, Menshykau D (2013) The control of branching morphogenesis. Open Biol 3(9):130088

Johnson R, Kirk P, Stumpf MPH (2014a) SYSBIONS: nested sampling for systems biology. Bioinformatics 31(4):604-605

Johnston ST, Simpson MJ, McElwain DLS, Binder BJ, Ross JV (2014) Interpreting scratch assays using pair density dynamics and approximate Bayesian computation. Open Biol 4(9):140097

Jones PJM, Sim A, Taylor HB, Bugeon L, Dallman MJ, Pereira B, Stumpf MPH, Liepe J (2015) Inference of random walk models to describe leukocyte migration. Phys Biol 12(6):066001

Kicheva A, Pantazis P, Bollenbach T, Kalaidzidis Y, Bittig T, Jülicher F, González-Gaitán M (2007) Kinetics of morphogen gradient formation. Science 315(5811):521-525

Kim Y, Stolarska MA, Othmer HG (2007) A hybrid model for tumor spheroid growth in vitro I: theoretical development and early results. Math Models Methods Appl Sci 17(supp01):1773-1798

Kondo S, Miura T (2010) Reaction-diffusion model as a framework for understanding biological pattern formation. Science 329(5999):1616-1620

Lee DT (1982) Medial axis transformation of a planar shape. IEEE Trans Pattern Anal Mach Intell 4(4):363369

Liepe J, Kirk P, Filippi S, Toni T, Barnes CP, Stumpf MPH (2014) A framework for parameter estimation and model selection from experimental data in systems biology using approximate Bayesian computation. Nat Protoc 9(2):439-456

Lindenmayer A (1968) Mathematical models for cellular interactions in development II. Simple and branching filaments with two-sided inputs. J Theor Biol 18(3):300-315 
Little MH (2015) Improving our resolution of kidney morphogenesis across time and space. Curr Opin Genet Dev 32:135-143

Little MH, McMahon AP (2012) Mammalian kidney development: principles, progress, and projections. Cold Spring Harb Perspect Biol. https://doi.org/10.1101/cshperspect.a008300

Macklin P, Edgerton ME, Thompson AM, Cristini V (2012) Patient-calibrated agent-based modelling of ductal carcinoma in situ (DCIS): from microscopic measurements to macroscopic predictions of clinical progression. J Theor Biol 301(May):122-140

Maeshima A, Sakurai H, Choi Y, Kitamura S, Vaughn DA, Tee JB, Nigam SK (2007) Glial cell-derived neurotrophic factor independent ureteric bud outgrowth from the Wolffian duct. J Am Soc Nephrol 18(12):3147-3155

Majumdar A, Vainio S, Kispert A, McMahon J, McMahon AP (2003) Wnt11 and Ret/Gdnf pathways cooperate in regulating ureteric branching during metanephric kidney development. Development 130(14):3175-3185

McLennan R, Dyson L, Prather KW, Morrison JA, Baker RE, Maini PK, Kulesa PM (2012) Multiscale mechanisms of cell migration during development: theory and experiment. Development 139(16):2935-2944

McLennan R, Schumacher LJ, Morrison JA, Teddy JM, Ridenour DA, Box AC, Semerad CL, Li H, McDowell W, Kay D, Maini PK, Baker RE, Kulesa PM (2015) Neural crest migration is driven by a few trailblazer cells with a unique molecular signature narrowly confined to the invasive front. Development 142(11):2014-2025

Menshykau D, Iber D (2013) Kidney branching morphogenesis under the control of a ligand-receptor-based Turing mechanism. Phys Biol 10(4):046003

Merks RMH, Koolwijk P (2009) Modeling morphogenesis in silico and in vitro: towards quantitative, predictive, cell-based modeling. Math Model Nat Phenom 4(4):149-171

Michos O, Cebrian C, Hyink D, Grieshammer U, Williams L, D’Agati V, Licht JD, Martin GR, Costantini F (2010) Kidney development in the absence of Gdnf and Spry1 requires Fgf10. PLoS Genet 6(1): 1000809

Miyazaki Y, Oshima K, Fogo A, Hogan BLM, Ichikawa I (2000) Bone morphogenetic protein 4 regulates the budding site and elongation of the mouse ureter. J Clin Investig 105(7):863-873

Murray JD, Oster GF, Harris AK (1983) A mechanical model for mesenchymal morphogenesis. J Math Biol 17(1):125-129

Ochoa-Espinosa A, Affolter M (2012) Branching morphogenesis: from cells to organs and back. Cold Spring Harb Perspect Biol. https://doi.org/10.1101/cshperspect.a008243

Ohta T, Mimura M, Kobayashi R (1989) Higher-dimensional localized patterns in excitable media. Physica D 34(1-2):115-144

Packard A, Georgas K, Michos O, Riccio P, Cebrian C, Combes AN, Ju A, Ferrer-Vaquer A, Hadjantonakis A-K, Zong H, Little MH, Costantini FD (2013) Luminal mitosis drives epithelial Cell dispersal within the branching ureteric bud. Dev Cell 27(3):319-330

Pathmanathan P, Cooper J, Fletcher A, Mirams G, Murray P, Osborne J, Pitt-Francis J, Walter A, Chapman SJ (2009) A computational study of discrete mechanical tissue models. Phys Biol 6(3):036001

Perfahl H, Hughes BD, Alarcón T, Maini PK, Lloyd MC, Reuss M, Byrne HM (2016) 3D hybrid modelling of vascular network formation. J Theor Biol 414:254-268

Qiao J, Nigam SK, Sakurai H (1999) Branching morphogenesis independent of mesenchymal-epithelial contact in the developing kidney. Proc Natl Acad Sci USA 96(13):7330-7335

Qiao J, Nigam SK, Bush KT, Steer DL, Stuart RO, Sakurai H, Wachsman W (2001) Multiple fibroblast growth factors support growth of the ureteric bud but have different effects on branching morphogenesis. Mech Dev 109(2):123-135

Rejniak KA, Anderson ARA (2011) Hybrid models of tumor growth. Wiley Interdiscip Rev Syst Biol Med 3(1):115-125

Riccio P, Cebrian C, Zong H, Hippenmeyer S, Costantini FD (2016) Ret and Etv4 promote directed movements of progenitor cells during renal branching morphogenesis. PLoS Biol 14(2):e1002382

Roeder I, Horn M, Glauche I, Hochhaus A, Mueller MC, Loeffler M (2006) Dynamic modeling of imatinibtreated chronic myeloid leukemia: functional insights and clinical implications. Nat Med 12(10):11811184

Schatten GP, Schnell S, Maini P, Newman SA, Newman T (2007) Multiscale modeling of developmental systems, vol 81. Academic Press, London 
Scialdone A, Mugford ST, Feike D, Skeffington A, Borrill P, Graf A, Smith AM, Howard M (2013) Arabidopsis plants perform arithmetic division to prevent starvation at night. Elife 2:e00669

Scott JG, Hjelmeland AB, Chinnaiyan P, Anderson ARA, Basanta D (2014) Microenvironmental variables must influence intrinsic phenotypic parameters of cancer stem cells to affect tumourigenicity. PLoS Comput Biol 10(1):e1003433

Serls AE, Doherty S, Parvatiyar P, Wells JM, Deutsch GH (2005) Different thresholds of fibroblast growth factors pattern the ventral foregut into liver and lung. Development 132(1):35-47

Shakya R, Watanabe T, Costantini FD (2005) The role of GDNF/Ret signaling in ureteric bud cell fate and branching morphogenesis. Dev Cell 8(1):65-74

Short K, Hodson M, Smyth I (2013) Spatial mapping and quantification of developmental branching morphogenesis. Development 140(2):471-478

Short KM, Combes AN, Lefevre J, Ju AL, Georgas KM, Lamberton T, Cairncross O, Rumballe BA, McMahon AP, Hamilton NA, Smyth IM, Little MH (2014) Global quantification of tissue dynamics in the developing mouse kidney. Dev Cell 29(2):188-202

Sottoriva A, Tavare S (2010) Integrating approximate bayesian computation with complex agent-Based models for cancer research. In: Proceedings of COMPSTAT'2010. Physica-Verlag, Heidelberg, pp 57-66

Srinivas S, Goldberg MR, Watanabe T, D’Agati V, Al Awqati Q, Costantini FD (1999) Expression of green fluorescent protein in the ureteric bud of transgenic mice: a new tool for the analysis of ureteric bud morphogenesis. Dev Genet 24(3-4):241-251

Sunnåker M, Busetto AG, Numminen E, Corander J, Foll M, Dessimoz C (2013) Approximate Bayesian computation. PLoS Comput Biol 9(1):e1002803

Tee JB, Choi Y, Shah MM, Dnyanmote A, Sweeney DE, Gallegos TF, Johkura K, Ito C, Bush KT, Nigam SK (2010) Protein kinase A regulates GDNF/RET-dependent but not GDNF/Ret-independent ureteric bud outgrowth from the Wolffian duct. Dev Biol 347(2):337-347

Tee JB, Choi Y, Dnyanmote A, Decambre M, Ito C, Bush KT, Nigam SK (2013) GDNF-independent ureteric budding: role of PI3K-independent activation of AKT and FOSB/JUN/AP-1 signaling. Biol Open 2(9):952-959

Toni T, Welch D, Strelkowa N, Ipsen A, Stumpf MPH (2009) Approximate Bayesian computation scheme for parameter inference and model selection in dynamical systems. J R Soc Interface 6(31):187-202

Turing AM (1952) The chemical basis of morphogenesis. Philos Trans R Soc B Biol Sci 237:37-72

Varner VD, Nelson CM (2014) Cellular and physical mechanisms of branching morphogenesis. Development 141(14):2750-2759

Vo BN, Drovandi CC, Pettitt AN, Simpson MJ (2015) Quantifying uncertainty in parameter estimates for stochastic models of collective cell spreading using approximate Bayesian computation. Math Biosci 263:133-142

von Neumann J (1966) Theory of self-reproducing automata. University of Illinois Press, Champaign

Watanabe T, Costantini FD (2004) Real-time analysis of ureteric bud branching morphogenesis in vitro. Dev Biol 271(1):98-108

Yu SR, Burkhardt M, Nowak M, Ries J, Petrášek Z, Scholpp S, Schwille P, Brand M (2009) Fgf8 morphogen gradient forms by a source-sink mechanism with freely diffusing molecules. Nature 461(7263):533536

Zubkov V, Combes AN, Short KM, Lefevre J, Hamilton NA, Smyth IM, Little MH, Byrne HM (2015) A spatially-averaged mathematical model of kidney branching morphogenesis. J Theor Biol 379:24-37 